\title{
On some aspects of platoon control problems
}

\author{
R.F. Curtain, O.V. Iftime and H.J. Zwart
}

\begin{abstract}
In Jovanavic and Bamieh [11] a comparison was made between the LQR control of a long, finite platoon and of an infinite version. They also "argue that the infinite platoons capture the essence of the large-but-finite platoons". We construct examples for which this does not happen. Hence the infinite-dimensional platoon model does not always serve as useful paradigm for the finite platoon model as it becomes increasingly long. It is clear that ones needs extra assumptions. In this paper we also provide some positive results by relating the Toeplitz approximations to circulant approximating systems.
\end{abstract}

\section{INTRODUCTION}

In Jovanavic and Bamieh [11] a comparison was made between the LQR control of a long, finite platoon and of an infinite version (which is easier to analyse mathematically). They pointed out the shortcomings of previous models in Levine and Athans [14], Melzer and Kuo [15], [16], which were due to lack of exponential stabilizability or detectabity of the infinite platoon model. Subsequently they proposed an alternative formulation and demonstrated that the infinite model reflected well the behaviour of the long finite platoon. Based on this and other examples they argue that "the infinite case is a useful paradigm to understand large platoons", but no theory to support this claim was given. In this paper we show by means of two counterexamples that finite and infinite platoon models can exhibit quite different behaviour. In view of the popularity in the literature of using spatially invariant models as a way of understanding the behaviour of finite platoon models, it is important to clarify which properties one is considering and whether or not the infinite model does serve as a useful indicator for these properties. Among the many properties one could consider of the LQR solution we focus on the following two properties of the closed-loop generator $A_{c l}$ : the growth bound $\omega_{c l}=$ $\sup \left\{\operatorname{Re} \lambda: \lambda \in \sigma\left(A_{c l}\right)\right\}$ and the transient bound $M$ which is such that for any $\omega>\omega_{c l}$ we have $\left\|e^{A_{c l} t}\right\| \leq M e^{\omega t}$.

The aim of this paper is to give an insightful analysis into the comparison of these properties of the LQR solution for a finite platoon model with its infinite version for the following

R.F. Curtain is with Department of Mathematics, University of Groningen, Postbus 800, 9700 AV Groningen, The Netherlands. R.F.Curtainerug.nl

O.V. Iftime is with the Department of Economics and Econometrics, University of Groningen, Nettelbosje 2, 9747 AE Groningen, The Netherlands, o.v.iftimeerug.nI

H.J. Zwart is with Department of Applied Mathematics, University of Twente, P.O. Box 217, 7500 AE Enschede, The Netherlands. h.j.zwart@math.utwente.nl class of scalar systems

$$
\begin{aligned}
& \dot{z}_{r}(t)=\sum_{l=-N}^{N} a_{l} z_{r-l}(t)+\sum_{l=-N}^{N} b_{l} u_{r-l}(t) \\
& y_{r}(t)=\sum_{l=-N}^{N} c_{l} z_{r-l}(t), \quad-N \leq r \leq N, \quad t \geq 0,
\end{aligned}
$$

where only finitely many of the coefficients $a_{l}, b_{l}, c_{l} \in \mathbb{C}$ (the set of complex numbers) are nonzero and $z_{k}, y_{k}$ and $u_{k}$ are set to zero for $|k|>N$. The above model of a long platoon can also be written in a compact form $\Sigma\left(\mathbf{A}_{N}, \mathbf{B}_{N}, \mathbf{C}_{N}, 0\right)$

$$
\begin{aligned}
& \dot{\mathbf{z}}_{N}(t)=\mathbf{A}_{N} \mathbf{z}_{N}(t)+\mathbf{B}_{N} \mathbf{u}_{N}(t), \\
& \mathbf{y}_{N}(t)=\mathbf{C}_{N} \mathbf{z}_{N}(t), \quad t \geq 0,
\end{aligned}
$$

where $\mathbf{u}, \mathbf{y}, \mathbf{z}$ are column vectors of size $2 N+1$, e.g., $\mathbf{z}_{N}(t)=$ $\left[\begin{array}{llll}z_{-N}(t) & z_{-N+1}(t) & \cdots & z_{N}(t)\end{array}\right]^{T}$ and $\mathbf{A}_{N}, \mathbf{B}_{N}, \mathbf{C}_{N}$ are $(2 N+1) \times(2 N+1)$ Toeplitz matrices. It is well known that large Toeplitz matrices have bad numerical properties and simulations are not in general a reliable way to investigate the properties of large-but-finite Toeplitz systems (see Böttcher and Silverman [3]). Consequently, it is important to analyze these systems analytically. The limit as $N \rightarrow \infty$ produces a system that is amenable to mathematical computations. This infinite-dimensional platoon model falls into the class of spatially invariant systems introduced in [1] and is given by

$$
\begin{aligned}
& \dot{z}_{r}(t)=\sum_{l=-\infty}^{\infty} a_{l} z_{r-l}(t)+\sum_{l=-\infty}^{\infty} b_{l} u_{r-l}(t), \\
& y_{r}(t)=\sum_{l=-\infty}^{\infty} c_{l} z_{r-l}(t), \quad r \in \mathbb{Z}, \quad t \geq 0,
\end{aligned}
$$

where $\mathbb{Z}$ is the set of integer numbers, $a_{l}, b_{l}, c_{l} \in \mathbb{C}$ and $z_{r}(t), u_{r}(t)$ and $y_{r}(t) \in \mathbb{C}$ are the state, the input and the output vectors, respectively, at time $t \geq 0$ and spatial point $r \in \mathbb{Z}$. As in [5], [7] we can formulate (3), (4) as a standard state linear system $\Sigma(A, B, C, 0)$

$$
\begin{aligned}
& \dot{z}(t)=(A z)(t)+(B u)(t), \\
& y(t)=(C z)(t), \quad t \geq 0,
\end{aligned}
$$

with the state space $Z$, the input space $U$ and the output space $Y$ equal to $\ell_{2}(\mathbb{C})=\left\{z \mid z=\left(z_{r}\right)_{r=-\infty}^{\infty}, z_{r} \in \mathbb{C}\right.$, $\left.\sum_{r=-\infty}^{\infty}\left|z_{r}\right|^{2}<\infty\right\}$. $A, B, C$ are convolution operators. This system has an infinite Toeplitz matrix representation. A more convenient representation is obtained by taking Fourier 
transforms $\check{x}=\mathfrak{F} x, A=\mathfrak{F}^{-1} \check{A} \mathfrak{F}$, so that

$$
((A \check{x})(t))\left(e^{\mathrm{j} \theta}\right)=\check{A}\left(e^{\mathrm{j} \theta}\right) \check{x}\left(e^{\mathrm{j} \theta}, t\right)=\left(\sum_{l=-\infty}^{\infty} a_{l} e^{\mathrm{j} \theta l}\right) \check{x}\left(e^{\mathrm{j} \theta}, t\right) .
$$

Note that our standing assumption is that only finitely many of the coefficients are nonzero which means that $\check{A}\left(e^{\mathrm{j} \theta}\right), \check{B}\left(e^{\mathrm{j} \theta}\right), \check{C}\left(e^{\mathrm{j} \theta}\right)$ are uniformly continuous in $\theta$ on $[0,2 \pi]$ and $\check{A}, \check{B}, \check{C} \in \mathbf{L}_{\infty}(\partial \mathbb{D} ; \mathbb{C})$, where $\partial \mathbb{D}$ denotes the unit circle. Hence $\check{A}, \check{B}, \check{C}$ define bounded operators on $\mathbf{L}_{2}(\partial \mathbb{D} ; \mathbb{C})$. Now, $\ell_{2}(\mathbb{C})$ is isometrically isomorphic to $\mathbf{L}_{2}(\partial \mathbb{D} ; \mathbb{C})$ under the Fourier transform $\mathfrak{F}\left(\|x\|_{\ell_{2}(\mathbb{C})}=\right.$ $\|\check{x}\|_{\mathbf{L}_{2}(\partial \mathbb{D}, \mathbb{C})}$ ). Hence $\|A\|=\|\check{A}\|_{\infty}$ (we refer to [7] for further details).

Taking Fourier transforms of the system equations (5), we obtain

$$
\begin{aligned}
& \dot{\check{z}}(t)=\mathfrak{F} \dot{z}(t)=\check{A} \check{z}(t)+\check{B} \check{u}(t), \\
& \check{y}(t)=\mathfrak{F} y(t)=\check{C} \check{z}(t), \quad t \geq 0,
\end{aligned}
$$

where $\check{A}=\mathfrak{F} A \mathfrak{F}^{-1}, \check{B}=\mathfrak{F} B \mathfrak{F}^{-1}$ and $\check{C}=\mathfrak{F} C \mathfrak{F}^{-1}$ are multiplicative operators.

The state linear system $\Sigma(A, B, C, 0)$ is isometrically isomorphic to the state linear system $\Sigma(\check{A}, \check{B}, \check{C}, 0)$ with the state space, input and output spaces $\mathbf{L}_{2}(\partial \mathbb{D} ; \mathbb{C})$. Their system theoretic properties are identical (see [4, Exercise 2.5]). For $\theta \in[0,2 \pi]$ the system (6) can be written as

$$
\begin{aligned}
& \dot{\check{z}}\left(e^{\mathrm{j} \theta}, t\right)=\check{A}\left(e^{\mathrm{j} \theta}\right) \check{z}\left(e^{\mathrm{j} \theta}, t\right)+\check{B}\left(e^{\mathrm{j} \theta}\right) \check{u}\left(e^{\mathrm{j} \theta}, t\right) \\
& \check{y}\left(e^{\mathrm{j} \theta}, t\right)=\check{C}\left(e^{\mathrm{j} \theta}\right) \check{z}\left(e^{\mathrm{j} \theta}, t\right), \quad t \geq 0 .
\end{aligned}
$$

In Section II we analyze the LQR control problem for two examples and show that both the growth bounds and the transient factors of the closed-loop operators for the finite and infinite platoon models are radically different. For one example the growth bounds satisfy $\omega_{N}<\omega_{\infty}$ and the transient factor increases without bound as $N \rightarrow \infty$, whereas for the other example $\omega_{N}>\omega_{\infty}$ and it has a transient bound of one.

In Section III our main results in Theorem 3.11 are that if $\Sigma(A, B, C, 0)$ is exponentially stabilizable and detectable and the Toeplitz approximating system $\Sigma\left(\mathbf{A}_{N}, \mathbf{B}_{N}, \mathbf{C}_{N}, 0\right)$ is uniformly stabilizable and detectable, then the solution to the approximating Riccati equations $Q_{N}$ converge strongly to $Q$, the solution to the infinite-dimensional one, as $N \rightarrow \infty$. However, lack of uniform stabilizability and detectability was insufficient to explain both counterexamples. Moreover, in many numerical examples of uniformly stabilizable and detectable approximating systems we obtained $\omega_{N}<\omega_{\infty}$. We showed that the Riccati solutions for the circulant matrix approximants to $\Sigma(A, B, C, 0)$ do exhibit very similar behaviour to the infinite-dimensional ones as $N \rightarrow \infty$. Only in the very special cases when only one of the system operators $\check{A}, \check{B}, \breve{C}$ depends on $\theta$ do we obtain the convergence of $\omega_{N}$ to $\omega_{\infty}$ and a transient bound independent of $N$. All results are illustrated by worked examples.

Conclusions are drawn in the last section and background results on Toeplitz and circulant matrices are collected in the Appendix.

\section{CountereXAmples}

In this section we argue that infinite platoons do not always capture the essence of the large-but-finite platoons. We analyze two examples for which the growth bounds of the LQR closed-loop finite and infinite platoon models are significantly different. The first example illustrates the difference in stability between a finite and an infinite platoon model.

Example 2.1: Consider the uncontrolled finite platoon model

$$
\begin{aligned}
\dot{z}_{r}(t) & =a_{0} z_{r}(t)+a_{1} z_{r-1}(t), \quad-N+1 \leq r \leq N \\
\dot{z}_{-N}(t) & =a_{0} z_{-N}(t), \quad t \geq 0
\end{aligned}
$$

with the system matrix

$$
\mathbf{A}_{N}=\left[\begin{array}{ccccccc}
a_{0} & 0 & 0 & 0 & \cdot & 0 & 0 \\
a_{1} & a_{0} & 0 & 0 & \cdot & 0 & 0 \\
0 & a_{1} & a_{0} & 0 & \cdot & 0 & 0 \\
\cdot & \cdot & \cdot & \cdot & \cdot & \cdot & \cdot \\
\cdot & \cdot & \cdot & \cdot & \cdot & \cdot & \cdot \\
0 & 0 & 0 & 0 & \cdot & a_{1} & a_{0}
\end{array}\right]
$$

$\mathbf{A}_{N}$ has the multiple eigenvalue $a_{0}$ and the growth bound $\omega_{N}=\max \left\{\operatorname{Re} \lambda: \lambda \in \sigma\left(\mathbf{A}_{N}\right)\right\}=a_{0}$. However the transient behaviour depends strongly on $N$. We make this explicit by decomposing $\mathbf{A}_{N}=a_{0} I_{N}+a_{1} F_{N}$, where $I_{N}$ is the $(2 N+$ $1) \times(2 N+1)$ identity matrix and $F_{N}$ is the $(2 N+1) \times$ $(2 N+1)$ nilpotent matrix with $F_{N}^{2 N+1}=0$. This gives

$$
e^{\mathbf{A}_{N} t}=e^{a_{0} t}\left(I_{N}+a_{1} t F_{N}+\ldots \frac{1}{(2 N) !}\left(a_{1} t\right)^{2 N} F_{N}^{2 N}\right) \text {. }
$$

Noting that $\left\|F_{N}\right\|=1$ we can obtain the estimates $\left\|e^{\mathbf{A}_{N} t}\right\| \leq$ $t^{2 N} e^{a_{0} t} e^{\left|a_{1}\right|}$ for $t \geq 1$ and for $\varepsilon>0$

$$
\begin{aligned}
& \left\|e^{\mathbf{A}_{N} t}\right\| \leq e^{\left(a_{0}+\left|a_{1}\right|\right) t}, t \geq 0 \\
& \left\|e^{\mathbf{A}_{N} t}\right\| \leq M(N) e^{\left(a_{0}+\varepsilon\right) t}, t \geq 0 .
\end{aligned}
$$

We now compare this with the infinite platoon model

$$
\dot{z}_{r}(t)=a_{0} z_{r}(t)+a_{1} z_{r-1}(t), \quad r \in \mathbb{Z}, \quad t \geq 0,
$$

which is isomorphic via Fourier transforms to the following system

$$
\dot{\tilde{z}}(t)=\left(a_{0}+a_{1} e^{-\mathrm{j} \theta}\right) \check{z}(t), \quad t \geq 0, \quad \theta \in[0,2 \pi] .
$$

The system matrix $\check{A}\left(e^{\mathrm{j} \theta}\right)=a_{0}+a_{1} e^{-\mathrm{j} \theta}$ has the continuous spectrum

$$
\sigma(\check{A})=\left\{(x, y) \mid \quad\left(x-a_{0}\right)^{2}+y^{2}=a_{1}^{2}\right\} .
$$

Thus

$$
\left\|e^{A t}\right\|=\left\|e^{\check{A} t}\right\|=\max _{\theta \in[0,2 \pi]}\left|e^{\left(a_{0}+a_{1} e^{-\mathrm{j} \theta}\right) t}\right|=e^{\left(a_{0}+\left|a_{1}\right|\right) t},
$$

and the growth bound $\omega_{\infty}=\sup \{\operatorname{Re}(\lambda): \lambda \in \sigma(A)\}=$ $a_{0}+\left|a_{1}\right|$, which is larger than $\omega_{N}=a_{0}$. The transient factor $M(N)$ increases as $N \rightarrow \infty$, whereas the transient factor of the infinite platoon model is one. Clearly the finite and infinite platoon models exhibit very different stability behaviour. If $a_{0}+\left|a_{1}\right|<0$ this example can serve as a (trivial) LQR example with $A$ and $\mathbf{A}_{N}$ representing the closed-loop operators.

The above example emphasizes that when comparing the behaviour of finite and infinite platoon models both the 
growth bound and the transient factor are important indicators. In the following example LQR example the transient factors are both 1 , but the growth bound of the finite platoon is larger than that of the infinite platoon model.

Example 2.2: Let $\beta>1$ be given. Consider the following finite platoon model of the form (1)

$$
\begin{aligned}
\dot{z}_{r}(t) & =z_{r}(t)+u_{r}(t)+\beta u_{r-1}(t),-N+1 \leq r \leq N \\
\dot{z}_{-N}(t) & =z_{-N}(t)+u_{-N}(t), \\
y_{r}(t) & =z_{r}(t), \quad-N \leq r \leq N, \quad t \geq 0 .
\end{aligned}
$$

which can be written in the compact form (2) with $\mathbf{A}_{N}=$ $\mathbf{C}_{N}=I_{N}$ and

$$
\mathbf{B}_{N}=\left[\begin{array}{ccccc}
1 & 0 & 0 & \ldots & 0 \\
\beta & 1 & 0 & \ldots & 0 \\
\ldots & \ldots & \ldots & \ldots & \ldots \\
0 & 0 & \ldots & \beta & 1
\end{array}\right]
$$

The finite platoon is obviously stabilizable and detectable for all $N$. Factorize $\mathbf{B}_{N} \mathbf{B}_{N}^{*}=W_{N} \operatorname{diag}\left(\beta_{k}(N)\right) W_{N}^{*}$, where $W_{N}$ is a $(2 N+1) \times(2 N+1)$ unitary matrix. Then the solution $\mathbf{Q}_{N}$ to the corresponding control Riccati equation is readily calculated $\mathbf{Q}_{N}=W_{N} \operatorname{diag}\left(\frac{1+\sqrt{1+\beta_{k}(N)}}{\beta_{k}(N)}\right) W_{N}^{*}$. Hence $\left\|\mathbf{Q}_{N}\right\|=\max _{k=0, \ldots, 2 N} \frac{1+\sqrt{1+\beta_{k}(N)}}{\beta_{k}(N)}$, which is achieved at $\beta_{\min }(N)$, the minimum value of $\beta_{k}(N)$. The closed-loop operator is given by

$$
\mathbf{A}_{N}-\mathbf{B}_{N}\left(\mathbf{B}_{N}\right)^{*} \mathbf{Q}_{N}=W_{N} \operatorname{diag}\left(-\sqrt{1+\beta_{k}(N)}\right) W_{N}^{*}
$$

Equalityence $\left\|e^{\left(\mathbf{A}_{N}-\mathbf{B}_{N}\left(\mathbf{B}_{N}\right)^{*} \mathbf{Q}_{N}\right) t}\right\|=e^{-\sqrt{1+\beta_{\min }(N)} t}$.

holds, We claim that for $\beta>1$ one eigenvalue of $\mathbf{B}_{N}\left(\mathbf{B}_{N}\right)^{*}$ HZ approaches 0 as $N \rightarrow \infty$. It is readily verified that $\mathbf{B}_{N} \mathbf{B}_{N}^{*} v_{N}=w_{N}$, where

$$
\begin{aligned}
& v_{N}=\left(-\beta^{-1}, \beta^{-2},-\beta^{-3}, \ldots, \beta^{-2 N},-\beta^{-2 N-1}\right)^{T}, \\
& w_{N}=\left(0,0,0, \ldots, 0,-\beta^{-2 N-1}\right)^{T} .
\end{aligned}
$$

Since $\beta>1$, one eigenvalue must become arbitrarily small as $N \rightarrow \infty$ which means that $\left\|\mathbf{Q}_{N}\right\| \rightarrow \infty$, and one eigenvalue of $\mathbf{A}_{N}-\mathbf{B}_{N} \mathbf{B}_{N}^{*} \mathbf{Q}_{N}$ approaches -1 as $N \rightarrow \infty$. Hence the growth bound $\omega_{N} \rightarrow-1$ as $N \rightarrow \infty$.

We show below that this behaviour is very different from that of the infinite platoon

$$
\begin{aligned}
& \dot{z}_{r}(t)=z_{r}(t)+u_{r}(t)+\beta u_{r-1}(t), \\
& y_{r}(t)=z_{r}(t), \quad r \in \mathbb{Z}, \quad t \geq 0 .
\end{aligned}
$$

This system is isomorphic via Fourier transforms to

$$
\begin{aligned}
& \dot{\dot{z}}(t)=\check{z}(t)+\left(1+\beta e^{-\mathrm{j} \theta}\right) \check{u}(t), \\
& \check{y}(t)=\check{z}(t), \quad t \geq 0, \quad \theta \in[0,2 \pi] .
\end{aligned}
$$

It is clearly exponentially detectable and it is exponentially stabilizable, since the matrix $\left[\lambda-1: 1+\beta e^{-\mathrm{j} \theta}\right]$ has rank one for all $\lambda \in \overline{\mathbb{C}}_{0}^{+}$and all $\theta \in[0,2 \pi]$ (see [5], [7]). The LQR Riccati equation $\check{Q}+\check{Q}-\check{Q}\left(1+\beta e^{-\mathrm{j} \theta}\right)\left(1+\beta e^{-\mathrm{j} \theta}\right)^{*} \check{Q}+1=0$ has the unique positive solution $\check{Q}\left(e^{\mathrm{j} \theta}\right)=\frac{1+\sqrt{2+\beta^{2}+2 \beta \cos \theta}}{1+\beta^{2}+2 \beta \cos \theta}$. with norm

$$
\|\check{Q}\|=\max _{0 \leq \theta \leq 2 \pi}\left\|\check{Q}\left(e^{\mathrm{j} \theta}\right)\right\|=\frac{1+\sqrt{1+(1-\beta)^{2}}}{(1-\beta)^{2}} .
$$

The closed-loop operator is $-\sqrt{2+\beta^{2}+2 \beta \cos \theta}$. Hence its growth bound $\omega_{\infty}=-\sqrt{2+\beta^{2}-2 \beta}<-1$ and its transient factor is 1 . Notice that $\omega_{\infty}$ decreases as $\beta$ increases. In contrast, for the finite platoon the growth bound satsifies $\omega_{N} \rightarrow-1$ as $N \rightarrow \infty$ for all $\beta>1$.

So for two examples we have shown that both the growth bounds and the transient factors can be radically different. The obvious conclusion is that the infinite-dimensional platoon is not always a useful paradigm for understanding the behaviour of large-but-finite platoon models as claimed in [11].

\section{MAIN RESUlTS}

In this section we give conditions under which the solution to the LQR problem for the infinite platoon will serve as a useful paradigm for the large, but finite platoon.

Using [4, Theorem 6.2.7],[5], [7, Theorems 4.1,4.2] and [13, Theorem 11.2.1] one can obtain the following result.

Theorem 3.1: The system $\Sigma(A, B, C, 0) \quad$ is exponentially stabilizable (detectable) if and only if $\left(\check{A}\left(e^{\mathrm{j} \theta}\right), \check{B}\left(e^{\mathrm{j} \theta}\right), \check{C}\left(e^{\mathrm{j} \theta}\right), 0\right)$ is stabilizable (detectable) for each $\theta \in[0,2 \pi]$. If the above holds, then the control Riccati equation for (3), (4)

$$
A^{*} Q+Q A-Q B B^{*} Q+C^{*} C=0,
$$

has a unique nonnegative solution $Q$ and $A_{Q}=A-B B^{*} Q$ generates an exponentially stable semigroup. Moreover, the control Riccati equation for (7)

$$
\check{A}^{*} \check{Q}+\check{Q} \check{A}-\check{Q} \check{B} \check{B}^{*} \check{Q}+\check{C}^{*} \check{C}=0 \text {, }
$$

has a unique nonnegative solution $\check{Q} \in \mathbf{L}_{\infty}(\partial \mathbb{D} ; \mathbb{C})$ and $\check{A}_{Q}=\check{A}-\check{B} \check{B}^{*} \check{Q}$ generates an exponentially stable semigroup. Furthermore, $\check{Q}\left(e^{\mathrm{j} \theta}\right)$ is continuous in $\theta$ on $[0,2 \pi]$.

Denote by $\pi^{N}: \mathbb{Z}=\ell_{2} \rightarrow \mathbb{C}^{2 N+1}$ the natural projection with $i^{N}: \mathbb{C}^{2 N+1} \rightarrow \ell_{2}$ the corresponding injection map: $\pi^{N} i^{N}=I_{2 N+1}$. Denote $Z^{N}:=\mathbb{C}^{2 N+1}$ with the induced inner product $\langle x, y\rangle_{N}=\left\langle i^{N} x, i^{N} y\right\rangle_{\ell_{2}}$. Then $\mathbf{A}_{N}, \mathbf{B}_{N}, \mathbf{C}_{N}$ are Toeplitz matrix representations of the maps $\left.\pi^{N} A\right|_{Z^{N}}$, $\left.\pi^{N} B\right|_{Z^{N}},\left.\pi^{N} C\right|_{Z^{N}}$, with $Z^{N}$ as the state space, input space and output space. For simplicity of notation we use the same notation for the maps as for the matrices. Hence the finite platoon system $\Sigma\left(\mathbf{A}_{N}, \mathbf{B}_{N}, \mathbf{C}_{N}, 0\right)$ converges strongly to the infinite-dimensional platoon system $\Sigma(A, B, C, 0)$ in the following sense.

$$
\begin{aligned}
e^{A t} z & =\lim _{N \rightarrow \infty} i^{N} e^{\mathbf{A}_{N} t} \pi^{N} z, \\
\left(e^{A t}\right)^{*} z & =\lim _{N \rightarrow \infty} i^{N} e^{\mathbf{A}_{N}^{*} t} \pi^{N} z, \forall z \in \ell_{2}
\end{aligned}
$$

uniformly on compact time intervals. Moreover, as $N \rightarrow \infty$ $i^{N} \mathbf{B}_{N} \pi^{N} u \rightarrow B u, i^{N} \mathbf{B}_{N}^{*} \pi^{N} z \rightarrow B^{*} z, i^{N} \mathbf{C}_{N} \pi^{N} z \rightarrow C z$, $i^{N} \mathbf{C}_{N}^{*} \pi^{N} y \rightarrow C^{*} y, i^{N} \pi^{N} z \rightarrow z$ for all $z, u, y \in \ell_{2}$. We also need following properties.

Definition 3.2: $\Sigma\left(\mathbf{A}_{N}, \mathbf{B}_{N}, \mathbf{C}_{N}, 0\right)$ are uniformly stabilizable and detectable if there exist $\mathbf{F}_{N}, \mathbf{L}_{N} \in$ $\mathbb{C}^{(2 N+1) \times(2 N+1)}, F, L \in \mathcal{L}\left(\ell_{2}\right)$ such that $i^{N} \mathbf{F}_{N} \pi^{N} z \rightarrow F z$, $i^{N} \mathbf{F}_{N}^{*} \pi^{N} z \rightarrow F^{*} z, i^{N} \mathbf{L}_{N} \pi^{N} z \rightarrow L z, i^{N} \mathbf{L}_{N}^{*} \pi^{N} z \rightarrow L z$ $\forall z \in \ell_{2}, A+B F$ and $A+L C$ generate exponentially stable semigroups, and there exist constants $M \geq 1, \beta>0$ 
such that for all $N \in \mathbb{N}\left\|e^{\left(\mathbf{A}_{N}+\mathbf{B}_{N} \mathbf{F}_{N}\right) t}\right\| \leq M e^{-\beta t}$ and $\left\|e^{\left(\mathbf{A}_{N}+\mathbf{L}_{N} \mathbf{C}_{N}\right) t}\right\| \leq M e^{-\beta t}, t \geq 0$.

An application of Kappel and Salamon [12, Theorem 1, Proposition 1] together with Ito [10] yield.

Theorem 3.3: Suppose that $\Sigma(A, B, C, 0)$ is exponentially stabilizable and detectable and the sequence of finitedimensional approximating systems $\Sigma\left(\mathbf{A}_{N}, \mathbf{B}_{N}, \mathbf{C}_{N}, 0\right)$ is uniformly stabilizable and detectable and converges strongly to $\Sigma(A, B, C, 0)$. For the state linear systems (5) and (2) let $Q \in \mathcal{L}\left(\ell_{2}\right)$ and $Q_{N} \in \mathcal{L}\left(Z^{N}\right)$ denote the unique nonnegative solutions of their respective Riccati equations (9) and

$$
\mathbf{A}_{N}^{*} Q_{N}+Q_{N} \mathbf{A}_{N}-Q_{N} \mathbf{B}_{N} \mathbf{B}_{N}^{*} Q_{N}+\mathbf{C}_{N}^{*} \mathbf{C}_{N}=0 . \text { (11) }
$$
Then $Q_{N}$ converges strongly to $Q$, i.e., $Q z=$ $\lim _{N \rightarrow \infty} i^{N} Q_{N} \pi^{N} z, \quad \forall z \in \ell_{2}$, and consequently $\left\|Q_{N}\right\|$ is uniformly bounded in $N$. Denote $A_{Q}:=A-B B^{*} Q$ and $A_{Q_{N}}:=\mathbf{A}_{N}-\mathbf{B}_{N} \mathbf{B}_{N}^{*} Q_{N}$. Then $A_{Q_{N}}$ converges strongly to $A_{Q}$, i.e., $i^{N} e^{A_{Q_{N}}{ }^{2}} \pi^{N} z \rightarrow e^{A_{Q} t} z, \forall z \in \ell_{2}$ as $N \rightarrow \infty$ uniformly on compact time intervals.

Moreover, there exist positive constants $\bar{M}, \mu$ such that

$$
\left\|e^{A_{Q} t}\right\| \leq \bar{M} e^{-\mu t}, \quad\left\|e^{A_{Q_{N}} t}\right\| \leq \bar{M} e^{-\mu t},
$$

for all $t \geq 0$, and for all $u \in \ell_{2}$ as $N \rightarrow \infty$, we have $\left\|C\left(\cdot I-A_{Q}\right)^{-1} B u-i^{N} \mathbf{C}_{N}\left(\cdot I_{N}-A_{N}\right)^{-1} \mathbf{B}_{N} \pi^{N} u\right\|_{\mathbf{H}_{2}} \rightarrow 0$ and $\left\|B^{*} Q\left(\cdot I-A_{Q}\right)^{-1} B u-i^{N} \mathbf{B}_{N}^{*} Q_{N}\left(\cdot I_{N}-A_{N}\right)^{-1} \mathbf{B}_{N} \pi^{N} u\right\|_{\mathbf{H}_{2}} \rightarrow$ 0 .

Note that the counterexample (4.1) in [12] shows that in general it is not true that $\| C\left(\cdot I-A_{Q}\right)^{-1} B u-i^{N} \mathbf{C}_{N}\left(\cdot I_{N}-\right.$ $\left.A_{Q_{N}}\right)^{-1} \mathbf{B}_{N} \pi_{N} u \|_{\infty} \rightarrow 0$ as $N \rightarrow \infty$. We remark that the solutions $Q_{N}$ of (11) are not Toeplitz in general.

Simple sufficient conditions for uniform stabilizability and detectability are given in the following lemma.

Lemma 3.4: 1) If $b_{0} \neq 0$ and $\left\|\check{B}-b_{0}\right\|_{\infty}<\left|b_{0}\right|$, then $\Sigma\left(\mathbf{A}_{N}, \mathbf{B}_{N}, 0,0\right)$ is uniformly stabilizable. Moreover, given $\beta>0$ there exists $F=\operatorname{diag}\left(f_{0}\right)$ such that

$$
\left\|e^{\left(\mathbf{A}_{N}+\mathbf{B}_{N} \mathbf{F}_{N}\right) t}\right\| \leq e^{-\beta t} .
$$

2) If $c_{0} \neq 0$, and $\left\|\check{C}-c_{0}\right\|<\left|c_{0}\right|$, then $\Sigma\left(\mathbf{A}_{N}, 0, \mathbf{C}_{N}, 0\right)$ is uniformly stabilizable. Moreover, given $\beta>0$ there exists $L=\operatorname{diag}\left(l_{0}\right)$ such that

$$
\left\|e^{\left(\mathbf{A}_{N}+\mathbf{L}_{N} \mathbf{C}_{N}\right) t}\right\| \leq e^{-\beta t} .
$$

3) $\Sigma\left(\mathbf{A}_{N}, \mathbf{B}_{N}, \mathbf{C}_{N}, 0\right)$ is uniformly stabilizable if there exists a nonzero $\delta$ such that $\lambda_{\min }\left(\mathbf{B}_{N} \mathbf{B}_{N}^{*}\right) \geq \delta^{2}$ for all $N$. Moreover the stability margin $\beta$ can be made arbitrarily large.

4) $\Sigma\left(\mathbf{A}_{N}, \mathbf{B}_{N}, \mathbf{C}_{N}, 0\right)$ is uniformly detectable if there exists a nonzero $\gamma$ such that $\lambda_{\min }\left(\mathbf{C}_{N}^{*} \mathbf{C}_{N}\right) \geq$ $\gamma^{2}$ for all $N$. Moreover the stability margin $\beta$ can be made arbitrarily large.

Strong convergence is insufficient to draw conclusions about the spectrum of $A_{Q_{N}}$. However, we note that in our Example 2.2 we only have uniform stabilizability if $\beta<1$. In this case we do not even have strong stability. If only one of the parameters $\check{A}, \check{B}, \check{C}$ depends on $\theta$, then we can prove better convergence results.

Lemma 3.5: In the case that $\check{A}=a_{0}, \check{B}=b_{0} \neq 0, \check{C} \neq 0$ there holds
1) $\lim \sup _{N \rightarrow \infty}\left\|\mathbf{Q}_{N}\right\|=\|Q\|$ and $\left\|e^{\mathbf{A}_{Q_{N}} t}\right\| \leq e^{-a_{0} t}$ for all $t \geq 0$.

2) If $\Sigma\left(\mathbf{A}_{N}, \mathbf{B}_{N}, \mathbf{C}_{N}, 0\right)$ is uniformly detectable, then $i^{N} \mathbf{Q}_{N} \pi^{N}$ converges strongly to $Q$ as $N \rightarrow \infty$ and for all $t \geq 0$ there holds

$$
\left\|e^{\mathbf{A}_{Q_{N}} t}\right\| \leq e^{\omega_{N} t}
$$

where $\omega_{N}$ is the growth bound of $e^{A_{Q_{N}} t}$.

In all simulations with systems satisfying the conditions of Lemma 3.5 we found that $\lim _{N \rightarrow \infty} \omega_{N}=\omega_{\infty}$. The same is true for systems satisfying the conditions of the next lemma.

Corollary 3.6: Suppose that $\check{A}=a_{0}, \check{C}=c_{0} \neq 0$.

1) $\Sigma\left(\mathbf{A}_{N}, \mathbf{B}_{N}, \mathbf{C}_{N}, 0\right)$ is uniformly stabilizable if and only if there exists a nonzero $\delta$ such that $\lambda_{\min }\left(\mathbf{B}_{N} \mathbf{B}_{N}^{*}\right)>\delta^{2}$ for all $N$.

2) If $\Sigma\left(\mathbf{A}_{N}, \mathbf{B}_{N}, \mathbf{C}_{N}, 0\right)$ is uniformly stabilizable, then then $i^{N} \mathbf{Q}_{N} \pi^{N}$ converges strongly to $Q$ as $N \rightarrow \infty$ and for all $t \geq 0$ there holds

$$
\left\|e^{\mathbf{A}_{Q_{N}} t}\right\| \leq e^{\omega_{N} t}
$$

where $\omega_{N}$ is the growth bound of $e^{A_{Q_{N}} t}$.

3) If $\Sigma\left(\mathbf{A}_{N}, \mathbf{B}_{N}, \mathbf{C}_{N}, 0\right)$ is not uniformly stabilizable, we still have

$$
\left\|e^{\mathbf{A}_{Q_{N}} t}\right\| \leq e^{-a_{0} t} \quad \forall t \geq 0 .
$$

The discretization of partial differential equations leads to systems with a real $\check{A}$ operator and constant $\check{B}, \check{C}$ operators (see El-Sayed and Krishnaprasad [8]). For such systems we also obtain nice convergence results.

Corollary 3.7: Suppose that $\check{A}$ is real, $\check{B}=b_{0} \neq 0$, $\check{C}=c_{0} \neq 0$. Then $\Sigma\left(\mathbf{A}_{N}, \mathbf{B}_{N}, \mathbf{C}_{N}, 0\right)$ is uniformly detectable and $i^{N} Q_{N} \pi^{N}$ converges strongly to $Q$ as $N \rightarrow \infty$ and $\lim _{N \rightarrow \infty}\left\|Q_{N}\right\|=\|Q\|$. The growth bound of $e^{A_{Q_{N}} t}$ converges to $\omega_{0}$ with $\left\|e^{A_{Q_{N}} t}\right\| \leq e^{\omega_{0} t}$.

Example 3.8: A spatial discretization of the bi-infinite heated rod

$$
\frac{\partial z}{\partial t}(t, x)=\alpha \frac{\partial^{2} z}{\partial x^{2}}(t, x)+u(t, x), x \in \mathbb{R}, \quad, t \geq 0 .
$$

with $z_{r}(t):=z(t, r \delta), u_{r}(t):=u(t, r \delta), r \in \mathbb{Z}$ leads to the spatially invariant system

$$
\dot{z}_{r}(t)=\alpha\left(z_{r-1}(t)-2 z_{r}(t)+z_{r+1}(t)\right)+u_{k}(t) .
$$

Where is $\delta$ ?

The solution to its Riccati equation is given by

$$
\check{Q}\left(e^{\mathrm{j} \theta}\right)=2 \alpha(\cos \theta-1)+\sqrt{4 \alpha^{2}(1-\cos \theta)^{2}+1},
$$

and the closed-loop operator

$$
\check{A}_{Q}\left(e^{\mathrm{j} \theta}\right)=-\sqrt{4 \alpha^{2}(1-\cos \theta)^{2}+1}
$$

has the growth bound of -1 . The corresponding Toeplitz approximating system has the solutions $Q_{N}=V_{N} D V_{N}^{*}$, where $V_{N}$ is an unitary matrix, $D=$ $\operatorname{diag}\left(2 \alpha\left(\cos \tau_{k}(N)-1\right)+\sqrt{4 \alpha^{2}\left(1-\cos \tau_{k}(N)\right)^{2}+1}\right)$, and $\tau_{k}(N)=\cos \frac{(k+1) \pi}{2 N+2}, k=0, \ldots, 2 N$. Moreover, the closed-loop operator

$$
A_{Q_{N}}=V_{N} \operatorname{diag}\left(-\sqrt{4 \alpha^{2}\left(1-\cos \tau_{k}(N)\right)^{2}+1}\right) V_{N}^{*},
$$


and the growth bound is $1-\sqrt{4 \alpha^{2}\left(1-\cos \frac{\pi}{2 N+2}\right)^{2}+1}$ which converges to -1 as $N \rightarrow \infty$.

Although we have given conditions for the strong convergence of $e^{\mathbf{A}_{Q_{N}} t}$ to $e^{A_{Q} t}$, this says nothing about the convergence of the stability margins. This point is clarified in Example 2.1.

In order to gain more information about the convergence of the stability margins we examine the related circulant approximants of $\check{A}, \check{B}, \breve{C}$ of dimension $n=2 N+1$ denoted by $\tilde{A}_{N}, \tilde{B}_{N}, \tilde{C}_{N}$ (see (15) in the Appendix).

Theorem 3.9: Consider the exponentially stabilizable and detectable system $\Sigma(A, B, C, 0)$ on the state-space $\ell_{2}$ with $Q$ the unique self-adjoint solution to the Riccati equation (9)

1) The following Riccati equation has a unique selfadjoint stabilizing solution $\tilde{Q}_{N}$ which is the circular approximant of $\mathscr{Q}$

$$
\tilde{A}_{N}^{*} \tilde{Q}_{N}+\tilde{Q}_{N} \tilde{A}_{N}-\tilde{Q}_{N} \tilde{B}_{N} \tilde{B}_{N}^{*} \tilde{Q}_{N}+\tilde{C}_{N}^{*} \tilde{C}_{N}=0 .
$$

$i^{N} \tilde{Q}_{N} \pi^{N}$ converges strongly to $Q$ and $i^{N} \tilde{A}_{Q_{N}} \pi^{N}$ converges strongly to $A_{Q}=A-B B^{*} Q$ as $N \rightarrow \infty$, where $\tilde{A}_{Q_{N}}=\tilde{A}_{N}-\tilde{B}_{N} \tilde{B}_{N}^{*} \tilde{Q}_{N}$ is a contraction semigroup.

2) $\lim \sup _{N \rightarrow \infty}\left\|\tilde{Q}_{N}\right\|=\|\check{Q}\|=\|Q\|$.

3) The growth bound $\tilde{\omega}_{N}$ of $e^{\tilde{A}_{Q_{N}} t}$ satisfies

$$
\tilde{\omega}_{N} \leq \omega_{\infty}, \quad \limsup _{N \rightarrow \infty} \tilde{\omega}_{N}=\omega_{\infty},
$$

where $\omega_{\infty}=\sup \left\{\operatorname{Re} \lambda, \lambda \in \sigma\left(A-B B^{*} Q\right)\right\}$, the growth bound of $e^{A_{Q} t}$. Moreover, for $N \in \mathbb{Z}$ we have

$$
\begin{gathered}
\left\|e^{\tilde{A}_{Q_{N}} t}\right\| \leq e^{\omega_{\infty} t} \quad \forall t \geq 0, \\
\left\|\left(\lambda I-\tilde{A}_{Q_{N}}\right)^{-1}\right\|_{\infty} \leq \frac{1}{\operatorname{Re} \lambda-\omega_{\infty}} \quad \text { for } \operatorname{Re} \lambda>\omega_{\infty} .
\end{gathered}
$$

4) For all $\lambda \in\left\{\operatorname{Re} \lambda>\omega_{\infty}\right\}$ we have

$$
\begin{array}{r}
\limsup _{N \rightarrow \infty}\left\|\left[\begin{array}{c}
\tilde{C}_{N} \\
\tilde{B}_{N}^{*} \tilde{Q}_{N}
\end{array}\right]\left(\lambda I_{N}-\tilde{A}_{Q_{N}}\right)^{-1} \tilde{B}_{N}\right\| \\
=\left\|\left[\begin{array}{c}
C \\
B^{*} Q
\end{array}\right]\left(\lambda I-A_{Q}\right)^{-1} B\right\| .
\end{array}
$$

Example 3.10: The circulant approximating system $\Sigma\left(\tilde{A}_{N}, \tilde{B}_{N}, \tilde{C}_{N}, 0\right)$ has $\tilde{A}_{N}=\tilde{C}_{N}=I_{2 N+1}$,

$$
\tilde{B}_{N}=\left[\begin{array}{ccccc}
1 & 0 & 0 & \ldots & \beta \\
\beta & 1 & 0 & \ldots & 0 \\
\ldots & \ldots & \ldots & \ldots & \ldots \\
0 & 0 & \ldots & \beta & 1
\end{array}\right] .
$$

It corresponds to the following (fictious) finite platoon model

$$
\begin{aligned}
\dot{z}_{r}(t) & =z_{r}(t)+u_{r}(t)+\beta u_{r-1}(t), \quad-N+1 \leq r \leq N \\
\dot{z}_{-N}(t) & =z_{-N}(t)+u_{-N}(t)+\beta u_{N}(t), \\
y_{r}(t) & =z_{r}(t), \quad-N \leq r \leq N, \quad t \geq 0 .
\end{aligned}
$$

Using the properties of circulant matrices from the Appendix, we factorize

$$
\tilde{B}_{N} \tilde{B}_{N}^{*}=U_{N} \operatorname{diag}\left(\mu_{k}(N)\right) U_{N}^{*}
$$

where the eigenvalues of $\tilde{B}_{N} \tilde{B}_{N}^{*}$ are $\mu_{k}(N)=1+\beta^{2}+$ $2 \beta \cos \frac{2 k \pi}{2 N+1}, k=0, \ldots, 2 N$ and the unitary matrix $U_{N}=$ $\frac{1}{\sqrt{2 N+1}}\left[e^{-\frac{2 \pi \mathrm{j} r s}{2 N+1}}\right]_{r, s=0, \ldots, 2 N}$. Hence we can derive the explicit solution to the corresponding circular Riccati equation

$$
\tilde{Q}_{N}=U_{N} \operatorname{diag}\left(\frac{1+\sqrt{1+\mu_{k}(N)}}{\mu_{k}(N)}\right) U_{N}^{*} .
$$

Hence

$$
\left\|\tilde{Q}_{N}\right\|=\frac{1+\sqrt{2+\beta^{2}-2 \beta \cos \frac{\pi}{2 N+1}}}{1+\beta^{2}-2 \beta \cos \frac{\pi}{2 N+1}} \rightarrow\|Q\|, N \rightarrow \infty .
$$

The closed-loop operator is given by

$$
\left(\tilde{A}_{N}\right)_{\tilde{Q}_{N}}=U_{N} \operatorname{diag}\left(-\sqrt{2+\beta^{2}+2 \beta \cos \frac{2 k \pi}{2 N+1}}\right) U_{N}^{*} .
$$

So the eigenvalues of the closed-loop circulant approximating system all lie in the spectrum of $A_{Q}$ and the growth bounds of their semigroups converge to $\omega_{\infty}=$ $-\sqrt{2+\beta^{2}-2 \beta}$ as $N \rightarrow \infty$

We now relate the solutions $\tilde{Q}_{N}$ of the circulant Riccati equation (13) to the solutions to (11). Note that we use the notation $|\cdot|_{N}$ instead of $|\cdot|_{2 N+1}$ for the weak norm defined in the appendix.

Theorem 3.11: Assume that $\Sigma(A, B, C, 0)$ is stabilizable and detectable and $\Sigma\left(\mathbf{A}_{N}, \mathbf{B}_{N}, \mathbf{C}_{N}, 0\right)$ is uniformly stabilizable and detectable. Then the following hold

1) $i^{N}\left(Q_{N}-\tilde{Q}_{N}\right) \pi^{N} \quad i^{N}\left(\mathbf{Q}_{N}-\tilde{Q}_{N}\right) \pi^{N}$, and $i^{N}\left(A_{Q_{N}}-\right.$ $\left.\tilde{A}_{Q_{N}}\right) \pi^{N}$ converge strongly to zero as $N \rightarrow \infty$.

2) $\left|Q_{N}-\tilde{Q}_{N}\right|_{N} \rightarrow 0$ and $\left|A_{Q_{N}}-\tilde{A}_{Q_{N}}\right|_{N} \rightarrow 0$ as $N \rightarrow 0$.

3) The closed-loop transfer functions $G_{N}^{c l}(\lambda)=$

$$
\begin{aligned}
& {\left[\begin{array}{c}
\mathbf{C}_{N} \\
\mathbf{B}_{N}^{*} Q_{N}
\end{array}\right]\left(\lambda I_{N}-A_{Q_{N}}\right)^{-1} \mathbf{B}_{N} \text { and } G^{c l}(\lambda)=} \\
& {\left[\begin{array}{c}
C \\
B^{*} Q
\end{array}\right]\left(\lambda I-A_{Q}\right)^{-1} B \text { satisfy }} \\
& \left\|\left|G^{c l}(\cdot)-G_{N}^{c l}(\cdot)\right|_{N}\right\|_{\mathbf{H}_{\infty}} \rightarrow 0 \text { and } \\
& \left\|\left|G^{c l}(\cdot)-G_{N}^{c l}(\cdot)\right|_{N}\right\|_{\mathbf{H}_{2}} \rightarrow 0 \text {. }
\end{aligned}
$$

4) $\lim _{N \rightarrow \infty} \frac{1}{2 N+1} \sum_{k=1}^{2 N} \lambda_{k}\left(Q_{N}\right)^{2}=\frac{1}{2 \pi} \int_{0}^{2 \pi} \check{Q}\left(e^{\mathrm{j} \theta}\right)^{2} \mathrm{~d} \theta$.

5) $\frac{1}{2 N+1} \sum_{k=1}^{2 N} \lambda_{k}\left(A_{Q_{N}}+A_{Q_{N}}^{*}\right)^{2} \rightarrow \frac{1}{2 \pi} \int_{0}^{2 \pi}\left(\check{A}_{Q}\left(e^{\mathrm{j} \theta}\right)+\right.$ $\left.\check{A}_{Q}\left(e^{\mathrm{j} \theta}\right)^{*}\right)^{2} \mathrm{~d} \theta$ for $N \rightarrow \infty$.

We remark that the convergence results for the transfer functions are necessarily weak. A simple calculation with the diagonal system with $\check{A}=a_{0}, \check{B}=b_{0}, \check{C}=c_{0}$ shows that we will never have $\left\|G^{c l}-i^{N} G_{N}^{c l} \pi^{N}\right\|_{\mathbf{H}_{\infty}} \rightarrow 0$ or $\left\|G^{c l}-i^{N} G_{N}^{c l} \pi^{N}\right\|_{\mathbf{H}_{2}} \rightarrow 0$. The most one could hope for is strong convergence $\left\|G^{c l} u-i^{N} G_{N}^{c l} \pi^{N} u\right\|_{\mathbf{H}_{\infty}} \rightarrow 0, \| G^{c l} u-$ $i^{N} G_{N}^{c l} \pi^{N} u \|_{\mathbf{H}_{2}} \rightarrow 0 \forall u \in U$. While the strong convergence in the $\mathbf{H}_{\mathbf{2}}$-norm does hold (see Theorem 3.3), the $\mathbf{H}_{\infty^{-}}$ norm convergence is unclear (see the counterexample (4.1) in [12]).

Theorem 3.11 explains the behaviour of Example 2.2 where $\omega_{N}>\omega_{\infty}$ for $\beta>1$; the system is not uniformly stabilizable. However, Example 2.1 shows that uniform stabilizability and detectability do not imply that $\lim \sup \omega_{N} \rightarrow$ $\omega_{\infty}$. For $a_{1} \geq 0$, we have $\omega_{N}=a_{0}<\omega_{\infty}=a_{0}+a_{1}$. This difference is explained by the transient bounds $M(N)$ that increase drastically with $N$ (see (8)). Similar gaps between $\omega_{N}$ and $\omega_{\infty}$ are found in numerical simulations. Does this 
mean that for this example the infinite-dimensional model cannot serve as a useful paradigm for the large finite platoon model? Another interpretation is that we should look at both the growth bound and the transient bound for a meaningful comparison. One well-defined measure that does this is the initial growth bound $\mu(A)$ of $e^{A t}$ defined in Hinrichsen and Pritchard [9, Definition 5.5.7].

$$
\mu(A)=\min \left\{\mu \in \mathbb{R} \mid\left\|e^{A t}\right\| \leq e^{\mu t} \quad \forall t \geq 0\right\} .
$$

This definition also makes sense for a bounded operator on a Hilbert space. It does depend on the norm used and for the uniform (spectral) norm it can be readily calculated from the formula $\mu(A)=\lambda_{\max }\left(A+A^{*}\right) / 2$. In all simulations we obtained $\lim \sup _{N \rightarrow \infty} \mu\left(A_{Q_{N}}\right)=\mu\left(\check{A}_{Q}\right)=\omega_{\infty}$ and we conjecture that this is indeed true in general. Unfortunately, all we can prove is the asymptotic average distribution of the eigenvalues in part 7 of Theorem 3.11.

One can also prove that similar results hold for $\mathbf{Q}_{N}$, the Toeplitz approximant of $\check{Q}$, which is not the same as $Q_{N}$.

\section{CONCLUSIONS AND FUTURE WORKS}

We have compared the growth bounds and the transient behaviour of the LQR closed-loop operators of scalar finite platoon models with their infinite versions. Simple examples showed that stabilizability and detectability are not sufficient to ensure similar stability behaviour of the LQR closed-loop platoon systems as $N \rightarrow \infty$. For the circulant approximating systems this does hold. Under the stronger conditions of uniform stabilizability and detectability of the finite platoon models we can show that the eigenvalues of the closedloop approximating systems have an average distribution that is asymptotic to that of the infinite-dimensional system. However, in general it is not true that the growth bound of the closed-loop finite platoon model $\omega_{N}$ converges to the growth bound $\omega_{\infty}$ of the closed-loop infinite platoon model. Only in the very special cases when just one of the system operators $\check{A}, \check{B}, \check{C}$ is not a constant do we obtain convergence of $\omega_{N}$ to $\omega_{\infty}$. Similar results are obtained for an alternative sequence of Toeplitz approximating systems.

Of course it is the MIMO case that is most interesting for applications, and this remains a challenging open problem. However, the scalar case has already demonstrated that LQR control of the infinite-dimensional platoon model does not always serve as a useful paradigm for the large finite platoon model.

\section{REFERENCES}

[1] Bamieh B. and Paganini F. and Dahleh M.A. Distributed control of spatially invariant systems, IEEE Trans. Automatic Control 47, 10911107, 2002.

[2] H.T. Banks and K. Kunisch. The Linear Regulator Problem for Parabolic Systems. SIAM. J. Control and Optim. 22, 684-698, 1984.

[3] A. Böttcher and B. Silvermann. Introduction to Large Truncated Toeplitz Matrices. Springer Verlag, New York, 1999.

[4] Curtain R.F. and Zwart H.J. An Introduction to Infinite-Dimensional Linear Systems Theory, Springer-Verlag, New York, (1995).

[5] Curtain R.F., Iftime O.V. and Zwart H.J. System Theoretic Properties of Platoon-Type Systems Proc. 47th IEEE Conference on Decision and Control, Cancun, Mexic, 1442-1447, 2008.
[6] R.M. Gray. Toeplitz and Circulant matrices: A review. Report 032, Stanford University Electronics Laboratory, Stanford California, 1971.

[7] Curtain R.F., Iftime O.V. and Zwart H.J. System theoretic properties of a class of spatially distributed systems Automatica, forthcoming, 2009.

[8] M L.El-Sayed and P.S. Krishnaprasad. Homogeneous interconnected systems: an example, IEEE Trans. Automatic Control 26: 894-901, 1981.

[9] D. Hinrichsen and A.J. Pritchard. Mathematical Systems Theory I: Modelling, State Space Analysis, Stability and Robustness. Springer Verlag, Berlin, 2000.

[10] Ito K. Strong convergence and convergence rates of approximating solutions for algebraic Riccati equations in Hilbert spaces, in Distributed Parameter Systems, Editors: W. Schappacher, F.Kappel and K.Kunisch. Springer Verlag, Berlin, 153-166, 1987.

[11] M.R. Jovanović and B. Bamieh. On the ill-posedness of certain vehicular platoon control problems. IEEE Trans. Automatic Control 50, 1307-1321, 2005.

[12] Kappel F. and Salamon D. An approximation theorem for the algebraic Riccati equation, SIAM Journal on Control and Optimization, 28, 1136-1147, 1990.

[13] Lancaster P. and Rodman L. Algebraic Riccati Equations, Oxford Science Publications, Clarendon Press, Oxford ,UK,(1995).

[14] W.S Levine and A. Athans. On the optimal error egulation of a string of moving vehicles. IEEE Trans. Automatic Control 11, 355-361, 1966.

[15] S.M. Melzer and B.C. Kuo. Optimal regulation of systems described by a countably infinite number of objects. Automatica 7, 359-366, 1971.

[16] S.M. Melzer and B.C. Kuo. A closed form solution for the optimal error regulation of a string of moving vehicles. IEEE Trans. Automatic Control 16, 50-52, 1971.

\section{APPEndiX: TOEPLitZ AND CiRCUlant MATRICES}

Denote by $\partial \mathbb{D}$ the unit circle. Consider a continuous scalar symbol $f \in \mathbf{L}_{2}(\partial \mathbb{D})$ given by $f\left(e^{\jmath \theta}\right)=\sum_{l=-\infty}^{\infty} f_{l} e^{-\jmath l \theta}$.

We denote the Toeplitz approximant matrix of order $n$ corresponding to $f$ by $\mathbf{T}_{n}(f)$

$$
\mathbf{T}_{n}(f)=\left[\begin{array}{ccccc}
f_{0} & f_{1} & f_{2} & \cdot & f_{n-1} \\
f_{-1} & f_{0} & f_{1} & \cdot & f_{n-2} \\
\cdot & \cdot & \cdot & \cdot & \cdot \\
\cdot & \cdot & \cdot & \cdot & \cdot \\
f_{-n+1} & f_{-n+2} & f_{-n+3} & \cdot & f_{0}
\end{array}\right]
$$

and the circulant approximant matrix of order $n$ by $\tilde{C}_{n}(f)$,

$$
\tilde{C}_{n}(f)=\left[\begin{array}{cccccc}
c_{0}^{(n)} & c_{1}^{(n)} & c_{2}^{(n)} & c_{3}^{(n)} & \cdot & c_{n-1}^{(n)} \\
c_{n-1}^{(n)} & c_{0}^{(n)} & c_{1}^{(n)} & c_{2}^{(n)} & \cdot & c_{n-2}^{(n)} \\
\cdot & \cdot & \cdot & \cdot & \cdot & \cdot \\
\cdot & \cdot & \cdot & \cdot & \cdot & \cdot \\
c_{1}^{(n)} & c_{2}^{(n)} & c_{3}^{(n)} & c_{4}^{(n)} & \cdot & c_{0}^{(n)}
\end{array}\right]
$$

where

$$
c_{k}^{(n)}=\frac{1}{n} \sum_{l=0}^{n-1} \tilde{f}\left(\frac{2 \pi l}{n}\right) e^{\frac{-2 \jmath \pi k l}{n}} .
$$

Circulant approximant matrices have very nice properties (see[6, Sections 3.1, 3.2] and the references therein).

In addition to the matrix spectral or induced $\mathbf{L}_{2}$-norm denoted by $\|\cdot\|$, following [6], we introduce the following n-norm for square matrices $M$ of order $n$

$$
|M|_{n}=\left(\frac{1}{n} \sum_{k=0}^{n-1} \sum_{l=0}^{n-1}\left|a_{k l}\right|^{2}\right)^{1 / 2}=\left(\frac{1}{n} \operatorname{trace}\left(M^{*} M\right)\right)^{1 / 2} .
$$

Some properties of this matrix norm we refer to [6, Lemma 2.3]. 